\title{
Falsely increased bispectral index score during deep hypothermic circulatory arrest in cardiac surgery
}

\author{
Jae Ouk Bang, Hyo Jung Son, Eun Ho Lee, Kyung-Don Hahm, and In-cheol Choi \\ Department of Anesthesiology and Pain Medicine, Asan Medical Center, University of Ulsan College of Medicine, Seoul, Korea
}

\begin{abstract}
The bispectral index score (BIS) is widely used as an accurate measure of the hypnotic effect of anesthetics and sedative drugs [1]. However, some clinical situations can lead to inaccuracy in its results [2]. We report two cases in which the BIS value constantly exceeded the expected levels during deep hypothermic circulatory arrest (DHCA) in cardiac surgery.

A 60-year-old male patient was diagnosed with a type III chronic aortic dissection accompanied by a thoracic aortic aneurysm and severe aortic valve regurgitation (AR). He was scheduled to undergo replacement of the descending thoracic aorta. He underwent Bentall surgery 1 year ago; however, this was complicated by third-degree atrioventricular block with an escape rate of less than 40 beats per minute. Therefore, he had a pacemaker implanted set in the DDD mode (Dual chamber pacing, Dual chamber sensing, Dual chamber function mode). The pacemaker mode was transformed to the DOO mode (Dual chamber pacing, No sensing, No function) before the induction because a fixed rate pacing mode such as the DOO mode can reduce the effect of electromagnetic interference.
\end{abstract}

Anesthesia was induced with $14 \mathrm{mg}$ etomidate, $50 \mathrm{mg}$ rocuronium, and continuous infusion of remifentanil and propofol. Intra-arterial catheters were inserted, and intubation was performed. We reduced the continuous infusion of rocuronium to $2 \mu \mathrm{g} / \mathrm{kg} / \mathrm{min}$ for adequate motor evoked potential (MEP) monitoring. A Swan-Ganz central venous catheter was placed in the left subclavian vein. Another central venous catheter was placed in the left internal jugular vein. Cerebrospinal fluid drainage was also performed at L3-4.

Before aortic cross clamping, the patient was cooled to a body temperature of $16.3^{\circ} \mathrm{C}$. At this time, the target value of BIS was between 0 and 10, although it rose greater than 70 despite the additional bolus injection of $5 \mathrm{mg}$ midazolam and the increase in the target effect site concentration of propofol (Fig. 1). The electromyography (EMG) graph did not indicate any significant activity $(<35 \mathrm{~dB})$. We began to suspect that the artifact had been caused by the patient's cardiac pacemaker, as it was observed without additional hypnotics. The DHCA was performed for 15 minutes, after which we raised the patient's body temperature to $27^{\circ} \mathrm{C}$. The BIS value fluctuated between 10 and 70 after DHCA. After weaning from cardiopulmonary bypass (CPB), the BIS value was maintained at a low level for 1 hour, after which it was elevated to 40 and kept between 40 and 50 until the end of the surgery. The postoperative course was uneventful, and the patient did not report awareness.

A 72-year-old male patient was diagnosed with a descending thoracic aortic aneurysm. He was scheduled to undergo replacement of both the distal arch and the descending thoracic aorta. Preoperative laboratory test results were within normal range.

The anesthetic preparation was identical to that used in case 1. However, as the MEP was not monitored well, we discontinued the continuous infusion of rocuronium.

Before aortic cross clamping, the patient was cooled to a body temperature of $17.2^{\circ} \mathrm{C}$. At this time, the BIS target value was between 0 and 10 . However, the BIS value abruptly increased to 75 , despite the additional bolus injection of 2 mg midazolam and the increase of the target effect site concentration of propofol. At this point, we noted that the EMG

Corresponding author: In-cheol Choi, M.D., Department of Anesthesiology and Pain Medicine, Asan Medical Center, University of Ulsan College of Medicine, 388-1, Pungnap 2-dong, Songpa-gu, Seoul 138-736, Korea. Tel: 82-2-3010-3862, Fax: 82-2-3010-6790, E-mail: icchoi@amc.seoul.kr (c) This is an open-access article distributed under the terms of the Creative Commons Attribution Non-Commercial License (http:// creativecommons.org/licenses/by-nc/3.0/), which permits unrestricted non-commercial use, distribution, and reproduction in any medium, provided the original work is properly cited. 


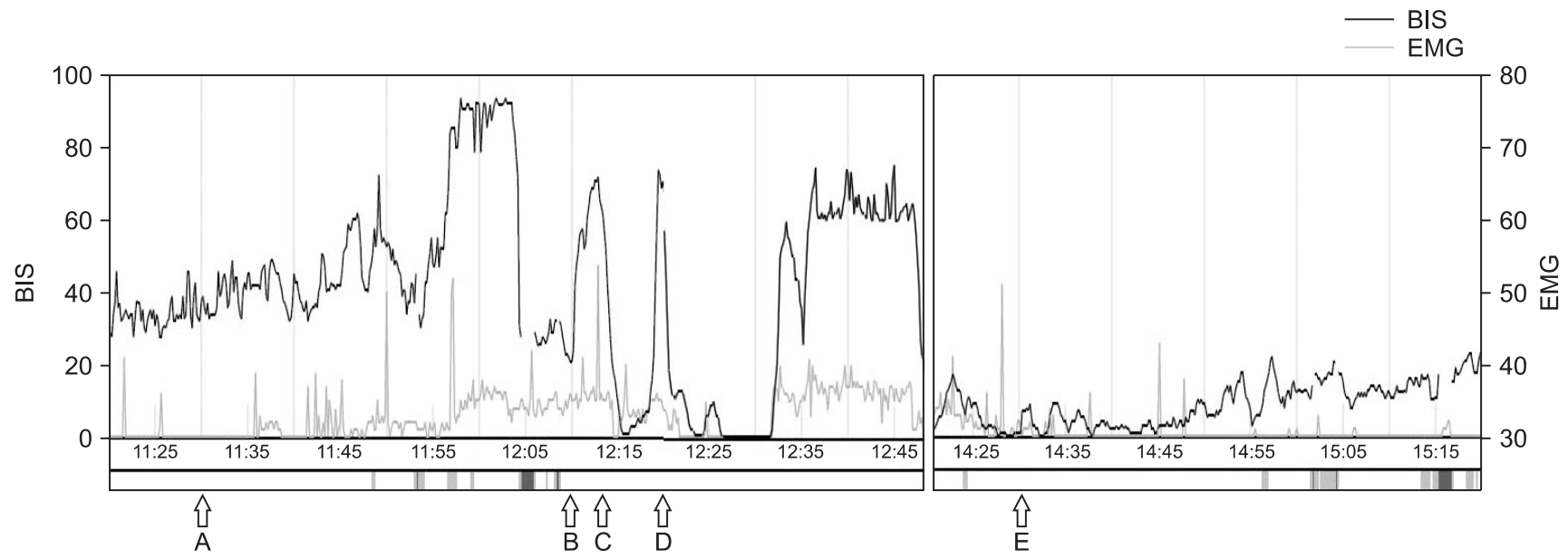

Fig. 1. Changes in BIS and EMG during surgery. A: Pump on; B: DHCA on; C: Midazolam bolus IV, increasing the continuous infusion of propofol; D: DHCA end; E: Pump off.

graph showed significant activity $(>35 \mathrm{~dB})$. We then gave a bolus injection of $20 \mathrm{mg}$ rocuronium. The EMG activity then declined, and the BIS value was maintained between 0 and 10 . We resumed the continuous infusion of rocuronium at a rate of $5 \mu \mathrm{g} / \mathrm{kg} / \mathrm{min}$, and the MEP slightly decreased. The DHCA was performed for 10 minutes, and the BIS value was maintained at 0 and 10 . Weaning from CPB was then successfully performed, but the BIS value was still maintained at a low level. The BIS value was slowly increased and maintained between 40 and 60 until the end of surgery. The postoperative course was uneventful. The patient did not report awareness.

The first case suggests that activated pacemakers interfere with the BIS sensor. In the absence of electroencephalography (EEG) signals, as in brain-dead subjects, or with extreme EEG suppression of deep hypothermia, the BIS algorithm is vulnerable to a wide range of signal artifacts [1]. Therefore, during complete isoelectric EEG suppression of DHCA, the BIS abruptly increased from 0 to $98-100$, thus indicating a radiofrequency pollution-like signal of the activated cardiac pacemaker [3].

The second case shows an unexpectedly high BIS value with high EMG activities. EMG activities are signal artifacts that occur within the frequency "range of interest" of the bispectrum because the EMG frequencies overlap the BIS algorithm's beta ratio of 30 - to $47-\mathrm{Hz}$ [1]. Therefore, a low frequency EMG signal can be interpreted as high frequency EEG by BIS, as it simulates the level of patient consciousness and the level of anesthesia [4]. In our case, as we did not use muscle relaxants due to MEP monitoring, we suspected that the unexpectedly high BIS value resulted from high EMG activity.
In conclusion, high EMG activity and electric device interference can create subtle signal noise without necessarily displaying recognized artifacts. This could then be misinterpreted by the BIS algorithm as EEG activity and assigned a falsely increased BIS value, although such a BIS value should be recognized by an anesthesiologist in order to avoid complications due to anesthetic overdose. Conversely, if the anesthesiologist is not certain of the cause of a high BIS value and questions whether the high BIS value is due to an artifact, intraoperative awakening and postoperative recall could occur. Therefore, anesthesiologists should observe their patients with extreme vigilance, and BIS monitoring should be seen as an additional method of general anesthesia monitoring and interpreted within the context of other clinical data and monitors.

\section{References}

1. Dahaba AA. Different conditions that could result in the bispectral index indicating an incorrect hypnotic state. Anesth Analg 2005; 101: 765-73

2. Vretzakis G, Dragoumanis C, Ferdi H, Papagiannopoulou P. Influence of an external pacemaker on bispectral index. Eur J Anaesthesiol 2005; 22: 70-2.

3. Mychaskiw G, Heath BJ, Eichhorn JH. Falsely elevated bispectral index during deep hypothermic circulatory arrest. Br J Anaesth 2000; 85: 798-800.

4. Sleigh JW, Steyn-Ross DA, Steyn-Ross ML, Williams ML, Smith P. Comparison of changes in electroencephalographic measures during induction of general anesthesia: influence of gamma frequency band and electromyogram signal. Br J Anaesth 2001; 86: 50-8. 